\title{
A SIMPLE SOLUTION FOR DIOPHANTINE EQUATIONS OF SECOND, THIRD AND FOURTH POWER
}

\section{A. K. Maran* \\ Scope of Research}

We know already that the set of positive integers, which are satisfying the Pythagoras equation of three variables and four variables are called Pythagorean triples and quadruples respectively. These are Diophantine equation of second power. The all unknowns in this Pythagorean equation have already been solved by mathematicians Euclid and Diophantine. However the solution defined by Euclid and Diophontine is also again having unknowns. The only possible to solve the Diophantine equations was trial and error method. Moreover, the trial and error method to obtain these values are not so practical and easy especially for time bound work, since the Diophantine equations are having more than two unknown variables. The scope of work is to solve the (1) Pythagorean triples,' (2) Pythagorean Quadruples ${ }^{2}$ and n-tuples and (3) Diophantine equations of third and fourth power ${ }^{3}$ by simple method. After conducting various exercises, the author has realized that there is a mathematical relation between the variables and he has attempted to establish the necessary formulae to solve these equations. These formulae and methods have been proved with appropriate examples. It is very useful for Students, Research scholars and persons those who are preparing question papers related to right-angled triangles, rectangular prisms and Number theory. ${ }^{4}$

* Assistant Executive Engineer (Vigilance), New Delhi. 


\section{The New Concept}

The new concept is that the mathematical relation between the variables in a Diophantine equation is that: If $x_{1}{ }^{m}+x_{2}{ }^{m}+x_{3}{ }^{m}+x_{4}{ }^{m}+\ldots \ldots+x_{n}{ }^{m}+y^{m}=z^{m}$ is written in the form $X+y^{m}=z^{m}$, one of the pair of the conjugate pair factors of $X$ is the key factor to solve the equation, where, $X=x_{1}{ }^{m}+x_{2}{ }^{m}+x_{3}{ }^{m}+\ldots . .+x_{n}{ }^{m}$

\section{Existing Methods}

For Diophantine equation of Pythagorean triples $x^{2}+y^{2}=r^{2}$, the solution defined by mathematicians Euclid and Diophantine as "All Pythagorean triples in which ' $x$ ' ' $y$ ', ' $r$ ' are without common factor and ' $x$ ' is odd are obtained by replacing the letters ' $a$ ' and ' $b$ ' in the triple $\left(a^{2}-b^{2}, 2 a b, a^{2}+b^{2}\right)$ by whole numbers that have odd sum and no common factor". For Diophantine equation of Pythagorean quadruples $x^{2}+y^{2}+z^{2}=r^{2}$, the solution defined by mathematicians Euclid and Diophantine as "All Pythagorean triples in which ' $x$ ', ' $y$ ', ' $r$ ' are without common factor and ' $x$ ' is odd are obtained by replacing the letters ' $a$ ', ' $b$ ', ' $c$ ' \& ' $d$ ' in the quadruple $\left(a^{2}-b^{2}+c^{2}-d^{2}, 2 b c+2 a d, a^{2}+b^{2}+c^{2}+d^{2}\right)$ by whole numbers that have odd sum and no common factor".

These equations having further unknown variables and it requires trial and error method. The Diophantine equation of $n$-tuples of second power, third power, fourth power etc., can be solved easily by using the following formulae $\&$ the methods.

\section{New Methods to Solve Diophantine Equations:}

\section{Procedure to solve the Diophantine equation of second power of n-tuples}

The Diophantine equation of $n$-tuples of second power is

$$
x_{1}{ }^{2}+x_{2}^{2}+x_{3}^{2}+x_{4}^{2}+\ldots . .+x_{n}^{2}+y^{2}=z^{2}
$$

Let, $\left[x_{1}^{2}+x_{2}^{2}+x_{3}^{2}+x_{4}^{2}+\ldots . .+x_{n}^{2}\right]=X$ and substituting in the above equation, we get

$$
x+y^{2}=z^{2}
$$

For given value of $x_{1}$, lef us assume any value for $x_{2}, x_{3}, x_{4}, \ldots . x_{n}$ (if these values are not given). So we have to find the value of $y$ and $z$ only. Identify all of the 
effective conjugate pair factors' $p \& q$ of $X$, but ' $p$ ' should be less than ' $q$ ' and also both $p \& q$ should be even or odd.

By substituting the effective set of values of $(p, q)$ in following formula, the value of ' $y$ ' \& ' $z$ ' can be determined.

$$
(y, z)=\left[\frac{q-p}{2}, \frac{q+p}{2}\right)
$$

Therefore the set of Pythagorean $n$-tuples are $\left\{x_{1}, x_{2}, x_{3^{\prime}}, x_{4}, \ldots . x_{n^{\prime}} y, z\right\}$

Some examples and its results are given in Table-1.

Table-1

\begin{tabular}{|c|c|c|c|c|c|c|c|c|c|c|}
\hline $\begin{array}{c}\text { Ex. } \\
\text { No }\end{array}$ & $\mathbf{x}_{\mathbf{1}}$ & $\mathbf{x}_{\mathbf{2}}$ & $\mathbf{x}_{\mathbf{3}}$ & $\mathbf{x}_{\mathbf{4}}$ & $\mathbf{x}_{\mathbf{5}}$ & $\mathbf{X}$ & $\mathbf{p}$ & $\mathbf{q}$ & $\mathbf{y}$ & $\mathbf{z}$ \\
\hline 1 & 24 & - & - & - & - & 576 & 6 & 96 & 45 & 51 \\
\hline 2 & 35 & - & - & - & - & 1225 & 7 & 175 & 84 & 91 \\
\hline 3 & 5 & 4 & - & - & - & 41 & 1 & 41 & 20 & 21 \\
\hline 3 & 4 & 8 & - & - & - & 80 & 2 & 40 & 19 & 21 \\
\hline 4 & 2 & 4 & 5 & - & - & 45 & 3 & 15 & 6 & 9 \\
\hline 6 & 3 & 4 & 6 & 8 & - & 125 & 5 & 25 & 10 & 15 \\
\hline
\end{tabular}

\section{Procedure to solve the Diophantine equation of third power of $n$-tuples}

The Diophantine equation of third power of any n-tuples is

$$
\left(x_{1}^{3}+x_{2}^{3}+x_{3}^{3}+x_{4}^{3}+\ldots . .+x_{n}^{3}\right)+y^{3}=z^{3}
$$

Let, $\left(x_{1}^{3}+x_{2}^{3}+x_{3}^{3}+\ldots . .+x_{n}^{3}\right)=X$ and substifuting in above equation, we get $x+y^{3}=z^{3}$

For given value of $x_{1}$, let us assume any value for $x_{2}, x_{3}, \ldots \ldots, x_{n}$, but $x_{1} \& x_{n} \neq 0$. The set of values of $(y, z)$ can be obtained from the following formula.

Refer new glossary of mathematical ferms in Appendix-1, which have been developed now by the outhor. 


$$
(y, z)=\left(\frac{\sqrt{12 q-3 p^{2}}-3 p}{6}, \frac{\sqrt{12 q-3 p^{2}}+3 p}{6}\right)
$$

where, $q$ is the factor of ' $X$ ', which satisties the following two conditions

(i) $\sqrt{12 q-3 p^{2}}$ is an integer.

(ii) $\mathrm{p}^{2}<\mathrm{q}$.

Some examples and its results are given in Table- 2 .

\section{Table-2}

\begin{tabular}{|c|c|c|c|c|c|c|c|c|c|c|}
\hline $\begin{array}{c}\text { e.g } \\
\text { No }\end{array}$ & $\mathbf{x}_{\mathbf{1}}$ & $\mathbf{x}_{\mathbf{2}}$ & $\mathbf{x}_{\mathbf{3}}$ & $\mathbf{x}_{\mathbf{4}}$ & $\mathbf{x}_{\mathbf{5}}$ & $\mathbf{X}$ & $\mathbf{p}$ & $\mathbf{q}$ & $\mathbf{y}$ & $\mathbf{z}$ \\
\hline 1 & 3 & 5 & - & - & - & 152 & 2 & 76 & 4 & 6 \\
\hline 2 & 6 & 8 & - & - & - & 728 & 8 & 91 & 1 & 9 \\
\hline 3 & 11 & 15 & - & - & - & 4706 & 2 & 2353 & 27 & 29 \\
\hline 4 & 5 & 7 & 9 & - & - & 1197 & 3 & 399 & 10 & 13 \\
\hline 5 & 11 & 12 & 13 & - & - & 5256 & 6 & 876 & 14 & 20 \\
\hline 6 & 1 & 3 & 4 & 5 & - & 217 & 1 & 217 & 8 & 9 \\
\hline 7 & 3 & 4 & 5 & 8 & - & 728 & 2 & 364 & 10 & 12 \\
\hline 8 & 1 & 5 & 6 & 7 & 8 & 1197 & 3 & 399 & 10 & 13 \\
\hline
\end{tabular}

\section{Procedure to solve the Diophantine equation of fourth power of $n$-tuples}

The Diophantine equation of third power of any n-tuples is

$$
\left(x_{1}{ }^{4}+x_{2}{ }^{4}+x_{3}{ }^{4}+x_{4}{ }^{4}+\ldots . .+x_{n}{ }^{4}\right)+y^{4}=z^{4}
$$

Let, $\left(x_{1}{ }^{4}+x_{2}{ }^{4}+x_{3}{ }^{4}+\ldots . .+x_{n}^{4}\right)=X$ and substituting in above equation, we get

$$
x+y^{4}=z^{4}
$$

For given value of $x_{1}$, let us assume any value for $x_{2}, x_{3}, \ldots \ldots, x_{n}$, but $x_{1} \& x_{n} \neq 0$. The set of values of $(y, z)$ can be obtained from the following formula.

$$
\{y, z\}=\{\sqrt{(q-p) / 2}, \sqrt{(q+p) / 2}\}
$$

Where, $p \& q$ are the conjugate pair factor of ' $X$ ', which satisfies the condition $\sqrt{(q-p) / 2} \& \sqrt{(q+p) / 2}$ are an integer and ' $p$ ' should be a square number. 
The different examples \& its results are given in Table- 3 :

Table-3

\begin{tabular}{|c|c|c|c|c|c|c|c|c|c|c|}
\hline $\begin{array}{c}\text { e.g } \\
\text { No }\end{array}$ & $\mathbf{x}_{1}$ & $\mathrm{x}_{2}$ & $\mathrm{x}_{\mathbf{3}}$ & $\mathrm{x}_{\mathbf{4}}$ & $\mathrm{x}_{\mathbf{5}}$ & $\mathbf{X}$ & $\mathbf{p}$ & $\mathbf{q}$ & $\mathbf{y}$ & $\mathbf{z}$ \\
\hline 1 & 2 & 2 & 3 & 4 & - & 369 & 9 & 41 & 4 & 5 \\
\hline 2 & 4 & 6 & 8 & 14 & - & 44064 & 144 & 306 & 9 & 15 \\
\hline 3 & 21 & 4 & 22 & 26 & - & 885969 & 441 & 2009 & 315 & 353 \\
\hline
\end{tabular}

\section{Conclusion}

The new method to solve the Diophantine equation of n-tuples of second power, third power, fourth power have been explained in this paper and which has been examined and proved in the chapter of Result \& discussion with appropriate examples. It is very useful for Students, Research scholars etc.

\section{References}

1. W.Gellert S.Gottwald, M.Hellwich. H.Kästner. H.Küstner, The VNR.

Concise Encyclopedia of Mathematics, Van Nostrand Reinhold, New York, 1989 p.672.

2. McGrow-Hill Encyclopedia of Science \& Technology, Vol-14, 9th Edition, New York, 2002, p.649.

3. hitp:// www.mathworld.wolfram.com

4. http:// www.mathworld.wolfram.com

\section{Appendix-1}

\section{Conjugate Pair Factors:}

The factor and corresponding quotient of any number are called conjugate pair factor of that number.

e.g: If $R=p \times q, p \& q$ are the conjugate pair factor of $R$. 